\title{
A New Protection Scheme for Fault Detection, Direction Discrimination, Classification, and Location in Transmission Lines
}

\author{
Joe-Air Jiang, Member, IEEE, Ching-Shan Chen, Student Member, IEEE, and Chih-Wen Liu, Member, IEEE
}

\begin{abstract}
This paper presents a new adaptive fault protection scheme for transmission lines using synchronized phasor measurements. The work includes fault detection, direction discrimination, classification, and location. Both fault-detection and fault-location indices are derived by using two-terminal synchronized measurements incorporated with distributed line model and modal transformation theory. The fault-detection index is composed of two complex phasors and the angle difference between the two phasors determines whether the fault is internal or external to the protected zone. The fault types can be classified by the modal faultdetection index. The proposed scheme also combines online parameter estimation to ensure protection scheme performance and achieve adaptive protection. Extensive simulation studies show that the proposed scheme provides a fast relay response and high accuracy in fault location under various system and fault conditions. The proposed method responds very well with regards to dependability, security, and sensitivity (high-resistance fault coverage).
\end{abstract}

Index Terms-Computer relaying, digital protection, global positioning system (GPS), phasor measurement unit (PMU), transmission-line protection.

\section{INTRODUCTION}

$\mathbf{T}$ RANSMISSION lines are the lifeblood of the power delivery. When a fault is detected, the protective relay must respond quickly to isolate the faulted line to preserve the stability of the rest of the system. Accurate estimates of the fault location are desirable for inspection, maintenance, and repair of the actual fault. Thus, the protective relaying and fault location of transmission lines are very important subjects.

Typically, distance protection is one common method to protect transmission lines. However, various conditions such as remote in-feed currents, fault-path resistance, and shunt capacitance, etc. degrade the performance. Recently, adaptive protection concepts were proposed to make relays adapt to changing system conditions and the performance of relays is to be improved [1]-[3].

The current differential protection scheme has been successfully applied to protect the entire line. However, the relay set-

Manuscript received November 29, 2001. This work was supported by the National Science Council under Grant NSC 91-2133-E-002-113.

J.-A. Jiang is with the Department of Bio-Industrial Mechatronics Engineering, National Taiwan University, Taipei 106, Taiwan, R.O.C.

C.-S. Chen and C.-W. Liu are with the Department of Electrical Engineering,

National Taiwan University, Taipei 106, Taiwan, R.O.C.

Digital Object Identifier 10.1109/TPWRD.2002.803726 tings are difficult to decide on due to the line-charging currents for long lines and the unobvious current variation during high resistance faults. Thus, the composite voltage and current measurements were used to improve relay sensitivity [4]. For fast fault clearance to improve system stability, the relaying schemes based on travelling wave are proposed [1], [5]. But, the techniques are difficult to detect close-in and voltage zero faults. Recently, fault-generated high-frequency transients were used to develop new relaying and fault-location schemes [6]. This method overcomes the drawback of the travelling-wave-based algorithm.

Due to the evolution of the high-speed microprocessor and digital communication technology, the computer-based measurement, protection, and control system have become common features of electric power substations [1]-[3]. Fiber-optic links are capable of transmitting information with a sufficient channel capacity and over a distance compatible with requirements of extra-high-voltage (EHV) transmission-line protection. Therefore, it is feasible to develop new communication-aided digital techniques for high-speed transmission-line protection. Better relaying and fault-location performance can be achieved using two-terminal synchronized measurements. Some algorithms based on global positioning system (GPS) synchronized measurements are proposed [6]-[10]. The synchronized sampling and phasor measurements for relaying and control are also described in [3]. Meanwhile, the IEEE has made the standard synchrophasors for power systems [11].

This paper is concerned with designing a new high-speed phasor measurement unit (PMU)-based protection scheme for EHV transmission lines. The fundamental principles are an extension of our previous work [9], [10]. The proposed multifunction scheme offers the speedy, robust, and accurate fault discrimination ability to protect the entire line. This method also provides accurate fault location. Simulation tests used to demonstrate the performance of the scheme are given in this paper.

\section{OPERATING PRINCIPLES}

The proposed scheme can achieve protective relaying tasks including fault detection, faulted-zone discrimination, as well as classification and fault location. The basic principles will be described. 


\section{A. Detection of Internal and External Faults}

In the early work [9], the authors use Clarke transformation in (1) to decouple the interphase quantities for a single-circuittransposed transmission line.

$$
[T]=\frac{1}{\sqrt{3}}\left[\begin{array}{ccc}
1 & \sqrt{2} & 0 \\
1 & \frac{-1}{\sqrt{2}} & \frac{\sqrt{3}}{\sqrt{2}} \\
1 & \frac{-1}{\sqrt{2}} & \frac{-\sqrt{3}}{\sqrt{2}}
\end{array}\right]
$$

An adaptive PMU-based fault-detection/location technique is developed using two-terminal synchronized phasors and distributed line model. The voltages at fault point $F$ (located at $x=D L \mathrm{~km}$ away from a receiving end, $D$ is the per-unit length, and $L$ is the total length of the protected line) and $x=0$ are taken as boundary conditions. The fault-detection indices are solved as

$$
\begin{aligned}
N_{m} & =\frac{1}{2}\left[V_{R m}-Z_{C m} I_{R m}\right] \\
& -\frac{1}{2} \exp \left[\Gamma_{m} L\right]\left[V_{S m}-Z_{C m} I_{S m}\right] \\
& =A_{m}-C_{m} \quad m=0, \alpha, \beta \\
M_{m} & =\frac{1}{2} \exp \left[-\Gamma_{m} L\right]\left[V_{S m}+Z_{C m} I_{S m}\right] \\
& -\frac{1}{2}\left[V_{R m}+Z_{C m} I_{R m}\right] \\
& =E_{m}-B_{m} \quad m=0, \alpha, \beta
\end{aligned}
$$

where $\Gamma_{m}$ represents modal propagation constant, $Z_{C m}$ is modal surge impedance, and $V_{R m}, V_{S m}, I_{R m}$, and $I_{S m}$ are the synchronized $m$-mode phasors of receiving-end/sending-end voltages and currents, respectively.

For untransposed lines, we can also find the transformation matrix to transform the coupled phase quantities to decoupled modal quantities with eigenvalue/eigenvector theory [12]. Thus, three modal fault detection indices can be also derived and whose form is the same as (2) under untransposed cases.

We adopt the moving-window recursive full-cycle discrete Fourier transform (DFT) to calculate fundamental phasors. The recursive formula for computing phasors is

$$
X_{i+1}=X_{i}+\frac{2}{N}[x(i+N)-x(i)] \exp \left[-j \frac{2 \pi}{N}\right]
$$

where

$$
\begin{array}{ll}
X & \text { phasor; } \\
i & \text { index for data window; } \\
x & \text { sampled data set; } \\
N & \text { sampling points per cycle. }
\end{array}
$$

The theoretical behavior of the modal fault-detection indices is discussed.

1) No-Fault Cases: In this case, $x(i+N)=x(i)$, the phasor $X$ is a constant phasor. The phase variation of phasor $X$ is $2 \pi / N$ for every forward step of moving window. Thus, the amplitudes of the four computed modal phasors $V_{R m}, V_{S m}, I_{R m}$, and $I_{S m}$ are all constant for healthy situation. The phase angles of these four phasors also rotate synchronously. It is easy to show that the $E_{m}$ and $B_{m}$ in (2-2) are equal since these four modal components satisfy the distributed transmission-line equation. This fact guarantees that $\left|M_{m}\right|$ (absolute value of $M_{m}$ ) for every moving data window is held at zero before fault occurrence. Similarly, $\left|N_{m}\right|$ is equal to zero too.

2) Internal or External Fault Cases: Once the post-fault data are included in moving window, $x(i+N) \neq x(i)$. From (3), we obtain that $X_{i+1} \neq X_{i}$. Thus, no matter what kinds of faults happen, the indices $\left|M_{m}\right|$ and $\left|N_{m}\right|$ will quickly deviate from zero value (which is monitored under the healthy case). In internal fault cases, the indices will rise to a steady value. However, for external faults, the indices will decay with time. So the detection indices $\left|M_{m}\right|$ and $\left|N_{m}\right|$ can identify the lines easily and properly as being faulted or unfaulted. The detection indices are very sensitive to faults even when high-resistance faults occur.

Consider the physical measurements due to current-transformer (CT) and capacitive-voltage-transformer (CVT) inaccuracies and numerical calculation errors, $\left|M_{m}\right|$ and $\left|N_{m}\right|$ may not be equal to zero. Therefore, a predetermined threshold is needed to avoid an error action.

The composite fault-detection index (i.e., $\left|M_{m}\right|$ or $\left|N_{m}\right|$ ), also can achieve direction discrimination and fault classification tasks discussed in the upcoming subsections. It is worthy to note, for example, that by only using a composite index $\left|M_{m}\right|$, the protective relaying function including fault detection, faulted-zone discrimination, and phase selection can be completed. Due to the high-speed response of the faultdetection index $\left|M_{m}\right|$, the tripping decision time of the relaying scheme is almost within half a cycle under various system and fault conditions. The overall performance of the scheme is shown in Section IV. Since the development of the scheme is based on the distributed line model, the proposed scheme is very suitable for protecting long lines.

\section{B. Fault-Direction Discrimination}

As mentioned before, the fault-detection index is composed of two complex phasors (i.e., $M_{m}=E_{m}-B_{m}=\left|E_{m}\right| \angle \theta_{E m}-$ $\left.\left|B_{m}\right| \angle \theta_{B m}\right)$. With the angle difference between the two phasors, we can attain faulted-zone discrimination. Take the index $M_{m}$, for example; the theory is discussed below.

1) No-Fault Cases: The directions of the currents are shown in Fig. 1(a). According to the basic principle mentioned before, we know $\left|E_{m}\right|=\left|B_{m}\right|$ and $\angle \theta_{E m}=\angle \theta_{B m}$, so that $\left|M_{m}\right|=0$.

2) Internal Fault Cases: As shown in Fig. 1(b), the current $I_{R}$ reverses and the index $\left|M_{m}\right|$ rises rapidly with a large slope. We obtain $\left|E_{m}\right| \neq\left|B_{m}\right|$ and $\angle \theta_{E m} \neq \angle \theta_{B m}$, so that $\left|M_{m}\right| \neq 0$.

3) External Fault Cases: This case can be described in two subcases.

a) Case 1: From Fig. 1(c), the directions of currents $I_{S}$ and $I_{R}$ are unchanged. In the steady state, $\left|E_{m}\right|=\left|B_{m}\right|$ and $\angle \theta_{E m}=\angle \theta_{B m}$, so that $\left|M_{m}\right|=0$ is the same as the no-fault cases.

b) Case 2: The directions of both current $I_{S}$ and $I_{R}$ reverse as shown in Fig. 1(d). Similarly, substituting these quantities into the transmission-line equation, we obtain $\left|E_{m}\right|=\left|B_{m}\right|$ and $\angle \theta_{E m}=\angle \theta_{B m}$, so that $\left|M_{m}\right|=0$.

For internal fault cases, the phase difference $\angle \theta_{E m}-\angle \theta_{B m}$ is theoretically held at constant in steady state. However, once 


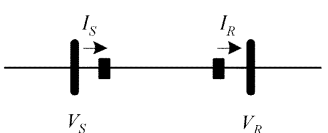

(a)

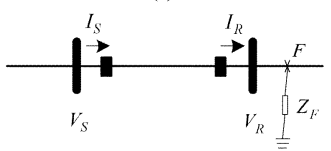

(c)

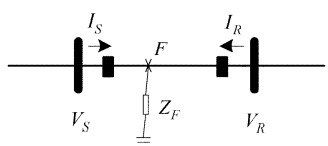

(b)

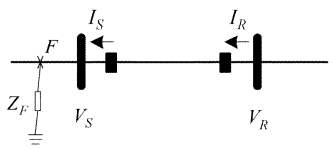

(d)

Fig. 1. Various situations of transmission lines: normal operation or faulted cases.

one of these two quantities rotates across the real axis of a complex plane (i.e., from the second to third quadrant or from the fourth to the first quadrant), depending on the definition of principal argument, the phase difference of these two phasors will automatically be compensated with $-2 \pi$. Therefore, the phase difference $\angle \theta_{E m}-\angle \theta_{B m}$ will no longer be a constant. For example, assume that the principal argument interval is $[-\pi$, $\pi]$ and at the $i$-th window $\angle \theta_{E m}(i)-\angle \theta_{B m}(i)=\angle \theta_{m \Delta}$, $\angle \theta_{m \Delta} \in(0, \pi)$. At the next window, if the phasor $E_{m}$ rotates some angle $\angle \theta_{m T}$ and falls into the third quadrant, where $\angle \theta_{E m}(i+1) \in(-\pi,-\pi / 2)$, the phase angle of the phasor $E_{m}$ will be corrected as $\angle \theta_{E m}(i+1)=\angle \theta_{E m}(i)+\angle \theta_{m T}-2 \pi$. But if the phasor $B_{m}$ is still located at the second quadrant so that the phase angle of $B_{m}$ is $\angle \theta_{B m}(i+1)=\angle \theta_{B m}(i)+\angle \theta_{m T}$. Then, $\angle \theta_{E m}(i+1)-\angle \theta_{B m}(i+1)=\angle \theta_{E m}(i)-\angle \theta_{B m}(i)-$ $2 \pi=\angle \theta_{m \Delta}-2 \pi \neq \angle \theta_{m \Delta}$. This means that the value of $\operatorname{abs}\left[\angle \theta_{E m}-\angle \theta_{B m}\right]$ is not a constant value and will vary linearly and periodically.

In conclusion, we cannot compute $\angle \theta_{E m}-\angle \theta_{B m}$ directly during implementation since the value of $\angle \theta_{E m}-\angle \theta_{B m}$ is not a constant in steady state. To cope with this difficulty, the authors use $\operatorname{abs}\left[\arg \left(E_{m} / B_{m}\right)\right]$ to compute the phase difference mentioned before. In steady state, the value of $\operatorname{abs}\left[\arg \left(E_{m} / B_{m}\right)\right]$ is held constant and does not vary with recursive moving window.

From the

$$
\begin{aligned}
\arg \left(\frac{E_{m}}{B_{m}}\right) & =\arg \left(\frac{\left|E_{m}\right| \angle \theta_{E m}}{\left|B_{m}\right| \angle \theta_{B m}}\right) \\
& =\angle \theta_{E m}-\angle \theta_{B m},
\end{aligned}
$$

the internal and external faults can be discriminated by the following criteria:

$$
\begin{aligned}
& \text { Internalfaults : } \operatorname{abs}\left[\arg \left(\frac{E_{m}}{B_{m}}\right)\right] \neq 0 \\
& \text { Externalfaults : } \operatorname{abs}\left[\arg \left(\frac{E_{m}}{B_{m}}\right)\right]=0
\end{aligned}
$$

where $\operatorname{abs}(\bullet)$ denotes absolute value and $\arg (\bullet)$ denotes phase angle. The proposed fault-direction discrimination criteria can be applied to transposed and untransposed lines.

The response of the discrimination criteria is fast and sensitive when the fault happens. For internal fault cases, the absolute value of the $\arg \left(E_{m} / B_{m}\right)$ rises to a certain value with a large slope. For external fault cases, the absolute value of the $\arg \left(E_{m} / B_{m}\right)$ will fast decay to zero. For the sake of high re-

\begin{tabular}{|c|c|c|c|c|c|c|c|c|c|c|c|c|}
\hline \multicolumn{2}{|c|}{ Fault types } & \multicolumn{3}{|c|}{$\mathrm{LG}$} & \multicolumn{3}{|c|}{ LLS } & \multicolumn{3}{|c|}{ LLG } & \multicolumn{2}{|c|}{$3 \mathrm{LS} / 3 \mathrm{LG}$} \\
\hline Basis & $\begin{array}{c}\text { Fault } \\
\text { detection } \\
\text { index }\end{array}$ & ag & bg & $\mathrm{cg}$ & abs & bcs & cas & $a b g$ & bcg & cag & abcs & abcg \\
\hline \multirow{3}{*}{$\begin{array}{c}\text { Phase } \\
\text { a }\end{array}$} & $\left|M_{a}^{0}\right|$ & 1 & 1 & 1 & 0 & 0 & 0 & 1 & 1 & 1 & 0 & 0 \\
\hline & $\left|M_{a}^{\alpha}\right|$ & 1 & 1 & 1 & 1 & 0 & 1 & 1 & 1 & 1 & 1 & 1 \\
\hline & $\left|M_{a}^{\beta}\right|$ & 0 & 1 & 1 & 1 & 1 & 1 & 1 & 1 & 1 & 1 & 1 \\
\hline \multirow{3}{*}{$\begin{array}{c}\text { Phase } \\
b\end{array}$} & $\left|M_{b}^{0}\right|$ & 1 & 1 & 1 & 0 & 0 & 0 & 1 & 1 & 1 & 0 & 0 \\
\hline & $\left|M_{b}^{\alpha}\right|$ & 1 & 1 & 1 & 1 & 1 & 0 & 1 & 1 & 1 & 1 & 1 \\
\hline & $\left|M_{b}^{\beta}\right|$ & 1 & 0 & 1 & 1 & 1 & 1 & 1 & 1 & 1 & 1 & 1 \\
\hline \multirow{3}{*}{$\begin{array}{c}\text { Phase } \\
\text { c }\end{array}$} & $\left|M_{c}^{0}\right|$ & 1 & 1 & 1 & 0 & 0 & 0 & 1 & 1 & 1 & 0 & 0 \\
\hline & $\left|M_{c}^{\alpha}\right|$ & 1 & 1 & 1 & 0 & 1 & 1 & 1 & 1 & 1 & 1 & 1 \\
\hline & $\left|M_{c}^{\beta}\right|$ & 1 & 1 & 0 & 1 & 1 & 1 & 1 & 1 & 1 & 1 & 1 \\
\hline
\end{tabular}
liability, we set a threshold incorporated with a counter limit to
TABLE I

AVAILABLE MODES UNDER DIFFERENT TRANSFORMATION BASIS

Letters ' $a$ ', ' $b$ ' and 'c' represent phase-a, phase- $b$, and phase-c, respectively. Letters ' $g$ ' and 's' represent 'ground fault' and 'short fault', respectively.

' 0 ' represents that the index value is less than the threshold and fails to detect faults. ' 1 ' represents that the index is greater than the threshold and rises with a large slope.

discriminate the faulted zone. The internal faults can be asserted when five consecutive samples of the absolute of $\arg \left(E_{m} / B_{m}\right)$ are over the predetermined threshold or restrains for external faults.

With regard to the threshold level, extensive EMTP studies have shown that the suitable threshold levels are $0.7 \mathrm{rad}$ for zero-mode signal and 0.2 rad for alpha and beta modes, respectively. These threshold values ensure that the relay is always stable for out-of-zone faults, without affecting its performance for internal faults.

It should be noted that the zero-mode signals are $E_{0}=B_{0}=0$ during healthy status in the three-phase balanced system. Thus, the $\arg \left(E_{0} / B_{0}\right)$ is an uncertain value. So the $\arg \left(E_{0} / B_{0}\right)$ is meaningless in the prefault period.

\section{Fault Classification and Phase Selection}

The algorithm of fault-type identification is slightly different for transposed and untransposed lines.

1) Algorithm for Transposed Lines: Not every modal component in (2) can be used for fault detection in transposed lines. With respect to different fault types, some modal components will disappear and cannot be used. The available modes based on Clarke transformation under various faults are shown in the enclosed area in Table I. The fault classification and phase selection cannot be achieved directly from the pattern listed in the enclosed area in Table I. Mansour and Swift [5] proposed a useful transformation technique such that three patterns are produced by Karrenbauer transformation to achieve fault classification and phase selection. This method also can be applied to Wedepohl and Clarke transformations. Our work is based on the Clarke transformation and collocates with the proposed faultdetection index $\left|M_{m}\right|$ to obtain three similar patterns (named as phase "a," "b," and "c" basis) shown in Table I. From Table I, the fault classification and phase-selection algorithm can be easily implemented by the logic circuit shown in Fig. 2. For example, if all logical signals of zero-mode $\left(\left|M_{a}^{0}\right|,\left|M_{b}^{0}\right|\right.$, and $\left.\left|M_{c}^{0}\right|\right)$ are 1 and the logical signal of beta-mode $\left|M_{a}^{\beta}\right|$ is 0 , only the logical 


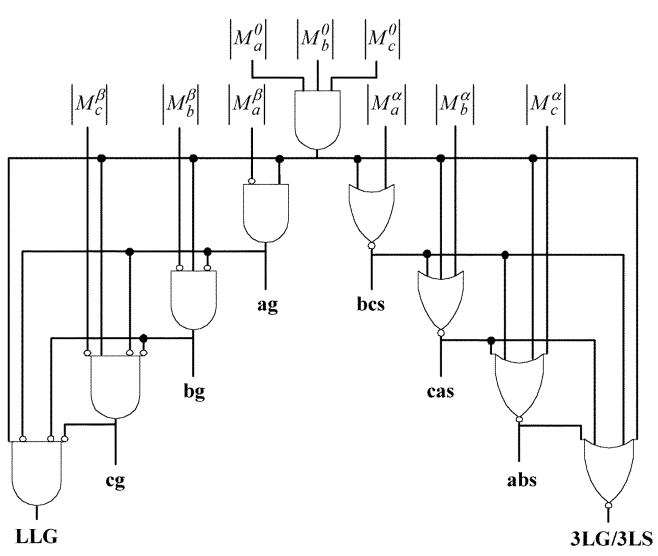

Fig. 2. Logic block of the fault classification and phase-selection algorithm.

output of "ag" is 1 and other logical outputs are zeros in Fig. 2. So the scheme classifies the fault as a-phase to ground fault.

There is a considerable variation between the " 0 " state and the " 1 " state of the computed index value in Table I. Therefore, reliable and fast fault-type identification can be performed. While the proposed faulted-phase selector is primarily based on power frequency measurements, the scheme is almost insensitive to various system and fault conditions. As shown in Fig. 2, the phase-selection algorithm can exactly identify the single-phase ground-fault cases to achieve single-pole tripping and initiate three-phase tripping for other fault types.

2) Algorithm for Untransposed Lines: For untransposed lines, all of the three modal fault-detection indices that are calculated with eigenvaluve/eigenvector theory can be used to detect faults. So, the fault-type identification cannot be achieved directly by the transformation technique described in 1). However, if the Clarke transformation matrix is used to transform the phase quantities to modal quantities for untransposed lines, there will be an unknown offset value for the modal fault-detection index under healthy conditions. Under fault conditions, the available modal fault-detection index will deviate from the offset value with a large slope but only cause small variations for unavailable mode signals.

Due to the unknown offset values, it is not easy to set a threshold value to detect faults for untransposed lines by using Clarke transformation. So the scheme uses the calculated transformation matrix described before (in Section II-A) to completely decouple interphase coupling effect and detect faults. Once the fault is correctly identified, we adopt the last offset value, which is generated by Clarke transformation, prior to the fault being detected as reference. And then the modified modal fault-detection indices are defined as

$$
\left|\bar{M}_{m}\right|=\frac{\left|M_{m}\right|}{\left|M_{m}\right|_{\text {prefault }}}
$$

where $\left|M_{m}\right|$ is the modal fault-detection index using Clarke transformation and $\left|M_{m}\right|_{\text {prefault }}$ is the last offset value before the fault is detected.

Therefore, the values of $\left|\bar{M}_{m}\right|$ are equal to 1 before the fault is detected. The $\left|\bar{M}_{m}\right|$ will deviate from 1 with a large slope for available modal signals and only cause a small variation of approximately 1 for unavailable modal signals. Using these modi-

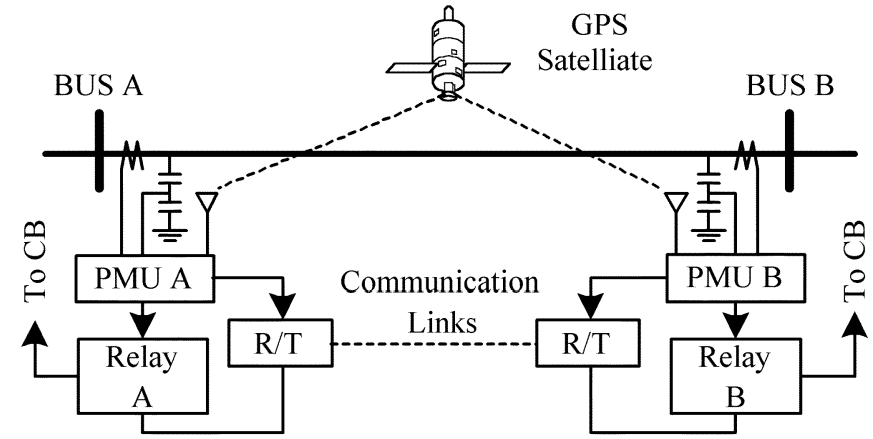

CB: Circuit Breaker, R: Receiver, T: Transmitter

Fig. 3. Block diagram of the proposed protection system.

fied modal fault-detection signals under a different transformation basis combined with a threshold level, the transformation patterns for untransposed lines will be the same as transposed cases. The fault classification and phase selection can also be completed.

\section{Fault Location}

The actual fault location can be accurately identified by the folowing formula [9]:

$$
D_{m}=\frac{\ln \left(\frac{N_{m}}{M_{m}}\right)}{2 \Gamma_{m} L} \quad m=0, \alpha, \beta .
$$

Based on two-terminal synchronized fundamental phasor measurements incorporated with the distributed line model, the proposed scheme offers high accuracy for fault location. Simulation results have verified that the performance of fault location has hardly been significantly affected by various system and fault conditions. The average fault-location error is well within $1 \%$. It should be noted that the formula in (6) still holds for untransposed lines.

\section{Protection System AND Algorithm}

The proposed protection system is shown in Fig. 3. The basic concept and algorithm of the scheme will be described briefly.

\section{A. Data Synchronization}

The three-phase phasors of voltage and current are measured simultaneously by PMUs at both ends of line. The global synchronism clock generator (GSCG) [10] has been built in the PMUs to provide an accurate and reliable external reference clock signal. The sampling synchronization of the PMU-GSCG configuration has been verified through field tests of Taipower 161-kV substations [10].

\section{B. Communication Links}

The format of transmitted synchronized data has been discussed in IEEE standard 1344-1995 [11]. The synchronized phasors of voltage and current are sent over high-speed communication links to the relays at the other ends of the protected line. With the rapid development of fiber-optic and digital-communication systems, multiplexed digital channels are often available for use with transmission-line protection. Therefore, high speed 


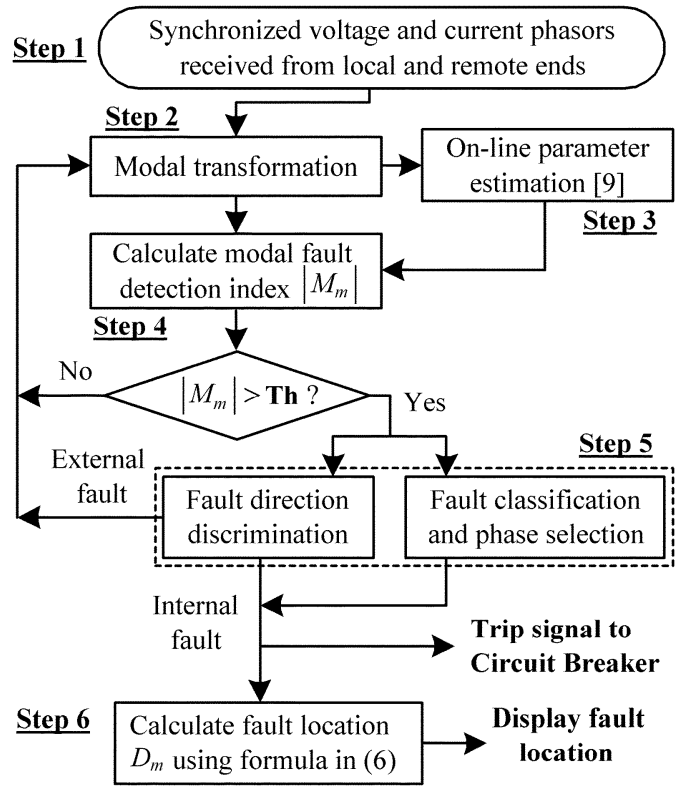

Fig. 4. Flowchart of the new adaptive protection scheme.

for data transfer can be achieved so that the transmission delay can be added only a few milliseconds to the tripping decision time of the protection scheme. Furthermore, the use of redundant communication links can ensure the overall reliability of the protection scheme.

\section{Protection Algorithm}

The flowchart of the adaptive protection algorithm is shown in Fig. 4 and is described as following

1) The relay receives the local PMU phasors and the synchronized data from the remote PMU through communication links.

2) The phasor quantities are decoupled into modal components using Clarke transformation matrix for transposed lines or decoupling matrix calculated by eigenvalue/eigenvector theory for untransposed lines. At this moment, the transformation is based on phase-a basis.

3) The online parameter estimation provides the online update of the line parameters at a predetermined interval. Hence, variation of line parameters will not affect the performance of the protection scheme. The performance of online parameter estimation for transposed lines has been verified in [10]. For untransposed cases, the line parameters also can be estimated using two-terminal phasor measurements.

4) The modal fault-detection index $\left|M_{m}\right|$ is calculated by using (2-2). When any available index $\left|M_{m}\right|$ is greater than the predetermined threshold (Th), the fault is detected.

5) At this stage, the protection scheme performs fault direction discrimination and fault classification simultaneously.

1) The modal signals, which detect a fault in step 4 , are used to discriminate fault direction. For the sake of high reliability, the scheme will check to see if there

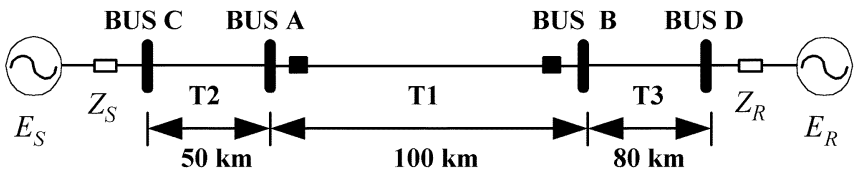

Fig. 5. One-line diagram of the simulated system.

is any modal signal that can be used to perform fault direction discrimination at the next window. Whether five consecutive $\operatorname{abs}\left[\arg \left(E_{m} / B_{m}\right)\right]$ data, whose transformation basis is phase-a, are greater than the predetermined threshold or not is used to distinguish internal faults from external faults. When an internal fault is confirmed, the protection scheme issues a trip signal to the circuit breaker. Combining a fault classification algorithm that will be described, if a single-phase-to-ground fault occurs, the scheme issues a single-pole tripping signal and initiates three-phase tripping for other types of fault. Otherwise, the algorithm will go back to step 2.

2) For fault-type identification, the fault-detection indices need to be further calculated by using Clarke transformations with respect to phase-b, and -c basis. Then, the scheme uses these generated detection indices incorporated with that of step 2 and the logical algorithm shown in Fig. 2 to perform the fault classification and faulted-phase selection tasks. Only slight modification for untransposed lines, the fault type can also be identified.

6) Finally, if an internal fault is confirmed, the protection scheme can also provide the fault location and display it to operators.

\section{Simulation Results AND Discussions}

In this paper, the EMTP/ATP simulator is adopted to evaluate the performance of the proposed scheme. Fig. 5 depicts the one-line diagram of the simulated system, which is a $345-\mathrm{kV}$ transposed transmission system. A distributed line model is used in this work. The transmission-line parameters and two Thévenin's equivalent source circuits are the same as in [10]. The phase difference between $E_{S}$ and $E_{R}$ is set as $20^{\circ}$. Practical considerations such as CTs, CVTs, and analog antialiasing filters (second-order low-pass Butterworth filters) with a cutoff frequency of $360 \mathrm{~Hz}$ are also implemented in the model. The sampling frequency is $1920 \mathrm{~Hz}$ (i.e., 32 samples/cycle). The fault inception angle is set to be $0^{\circ}$ with respect to a-phase voltage waveform. $\mathrm{T} 1$ is a protected line segment. $\mathrm{T} 2$ and $\mathrm{T} 3$ are used to evaluate the scheme's performance under external faults.

To demonstrate the robustness of the protection scheme, the simulations are conducted with respect to various system and fault conditions. The symbols $T_{f}, T_{d}$, and $T_{t}$ are defined as fault inception time, fault-detection time, and tripping decision time, respectively. The selected simulation results are briefly summarized as follows. 

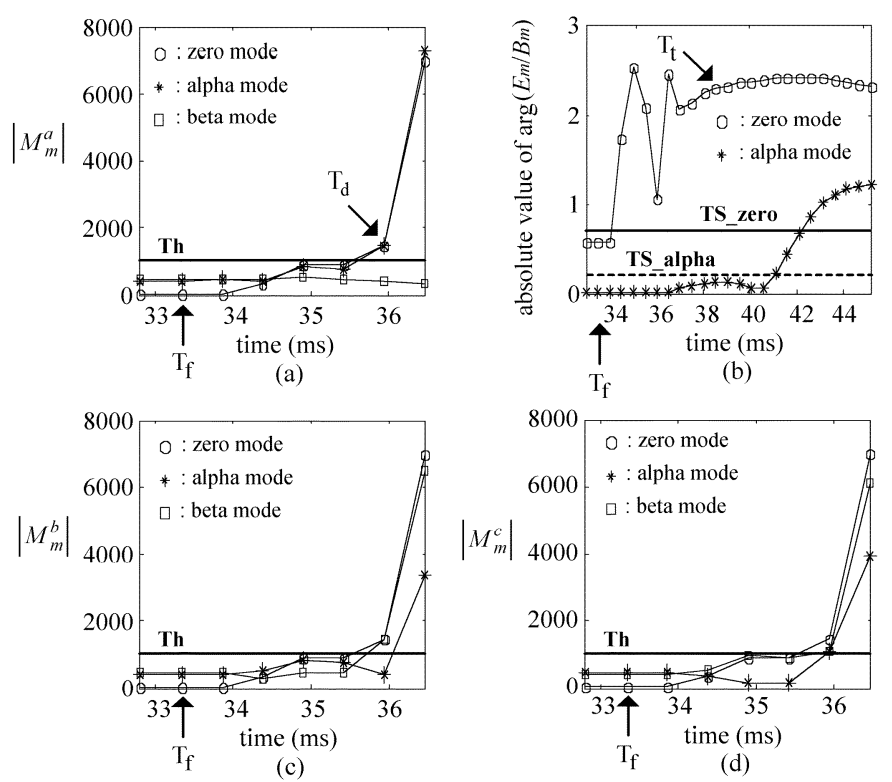

Fig. 6. Relay response for an internal a-g fault. (a) The behaviors of modal fault-detection index $\left|M_{m}\right|$ under phase-a basis. (b) The response curves of $\operatorname{abs}\left[\arg \left(E_{m} / B_{m}\right)\right] .(\mathrm{c})$ and (d) are the behaviors of modal fault-detection index under phase-b and -c basis, respectively.

\section{A. Typical Responses for Internal Faults}

Fig. 6 shows the responses of the relaying scheme to an a-phase to ground fault. The fault path resistance is $1 \Omega$ and fault location is set at 0.1 p.u. The response curves of modal fault-detection index under the phase-a basis are given in Fig. 6(a). It indicates that zero-mode and alpha-mode signals are greater than the predefined threshold level $(\mathbf{T h}=1000)$ at the time $2.604 \mathrm{~ms}$ after a fault occurrence such that the internal fault is detected. So these two modal signals can be used to discriminate fault direction. Fig. 6(b) shows the responses of abs[angle $\left.\left(E_{m} / B_{m}\right)\right]$. The threshold settings are $\mathbf{T S}$ zero $=0.7 \mathrm{rad}$ for zero-mode signal and TS $\_$alpha $=$TS_beta $=0.2$ rad for alpha and beta modes, respectively. An inspection of this figure clearly observes that the zero-mode signal is well above the predefined threshold in five consecutive samples. Thus, the proposed relaying scheme confirms a tripping decision at the time $5.208 \mathrm{~ms}$ after fault occurrence. Fig. 6(c) and (d) shows responses of modal fault-detection index under phase-b and -c basis, respectively. Using Fig. 6(a), (c), and (d) incorporated with the fault classification logic shown in Fig. 2, the scheme also correctly identifies the faulted phase (a-phase) when the internal is confirmed.

However, it should be noted that this time does not include any delays due to the data-transmission delay and calculation times, etc. In practice, these may add a few milliseconds to the overall tripping decision time.

The criterion used for performance evaluation of fault location error is defined as

$$
\begin{aligned}
& \operatorname{error}(\%) \\
= & \mid \operatorname{actual} \text { location }(\text { p.u. }) \text {-calculated location }(\text { p.u. }) \mid \\
& * 100 .
\end{aligned}
$$
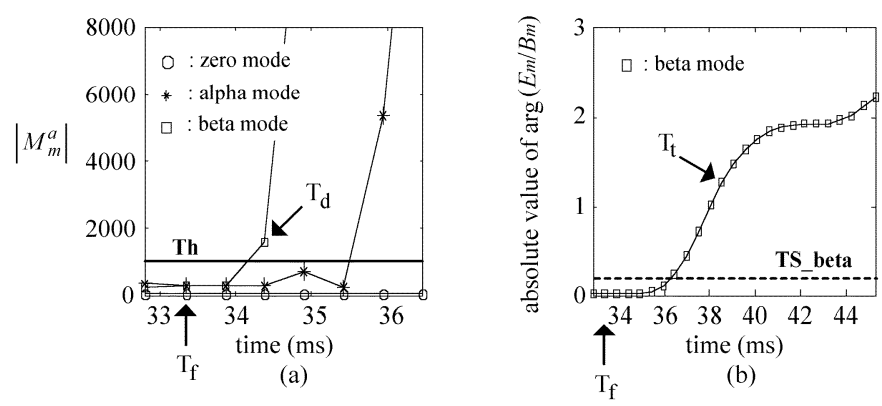

Fig. 7. Relay response for an internal a-b-c-g fault. (a) The behaviors of modal fault-detection index $\left|M_{m}\right|$ under phase-a basis. (b) The response curves of $\operatorname{abs}\left[\arg \left(E_{m} / B_{m}\right)\right]$
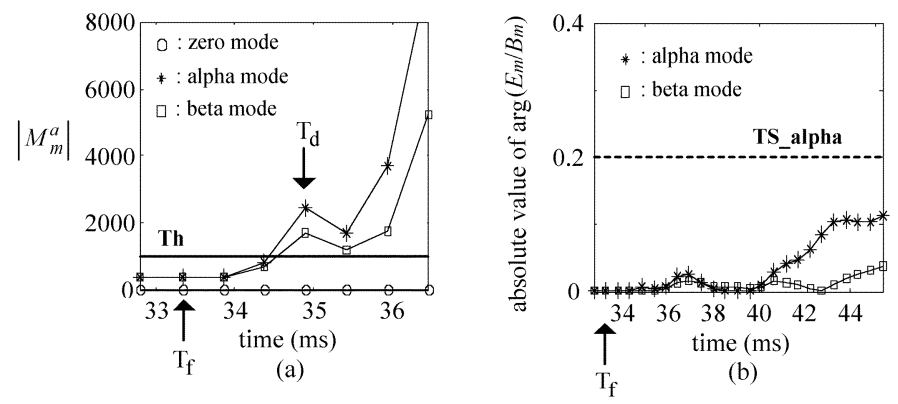

Fig. 8. Relay response for an external c-a-s fault. (a) The behaviors of modal fault-detection index under phase-a basis. (b) The response curves of $\operatorname{abs}\left[\arg \left(E_{m} / B_{m}\right)\right]$.

For the internal fault mentioned before, the fault location error of zero mode and alpha mode at the end of the third cycle after fault inception are 0.397 and $0.250 \%$, respectively.

There is no obvious characteristic difference between the faulted and healthy phases under high impedance fault (HIF). So the conventional relays are difficult to detect HIF. The proposed scheme can cope with this difficulty. To demonstrate the sensitivity of the relaying scheme, a three-phase-to-ground fault is selected as a simulation case whose fault-path resistance is $1 \mathrm{k} \Omega$ and fault location is set at 0.5 p.u. As shown in Fig. 7(a), the beta-mode fault-detection index will be first greater than the threshold so that the internal fault is detected quickly. At the next data window, the alpha-mode signal is not greater than the predefined threshold yet. So only the beta-mode signal is used to discriminate between internal and external faults. Fig. 7(b) shows that the proposed scheme will issue a tripping decision signal at the time of $5.208 \mathrm{~ms}$ after fault inception. Even if the fault resistance is up to $1 \mathrm{k} \Omega$, the relaying scheme still provides excellent performance. For the high-impedance fault, the fault-location error of alpha mode and beta mode are 0.770 and $0.834 \%$, respectively.

From the two cases presented before, it is clearly observed that the tripping decision time of the proposed scheme is well within half a cycle.

\section{B. Typical Responses for External Faults}

Fig. 8 shows the relay response for a c-a phase short fault occurring in line segment T3. The fault location is set at 0.8 p.u. away from bus D. An inspection of Fig. 8(a) clearly observes that the alpha-mode and beta-mode fault-detection indices are 

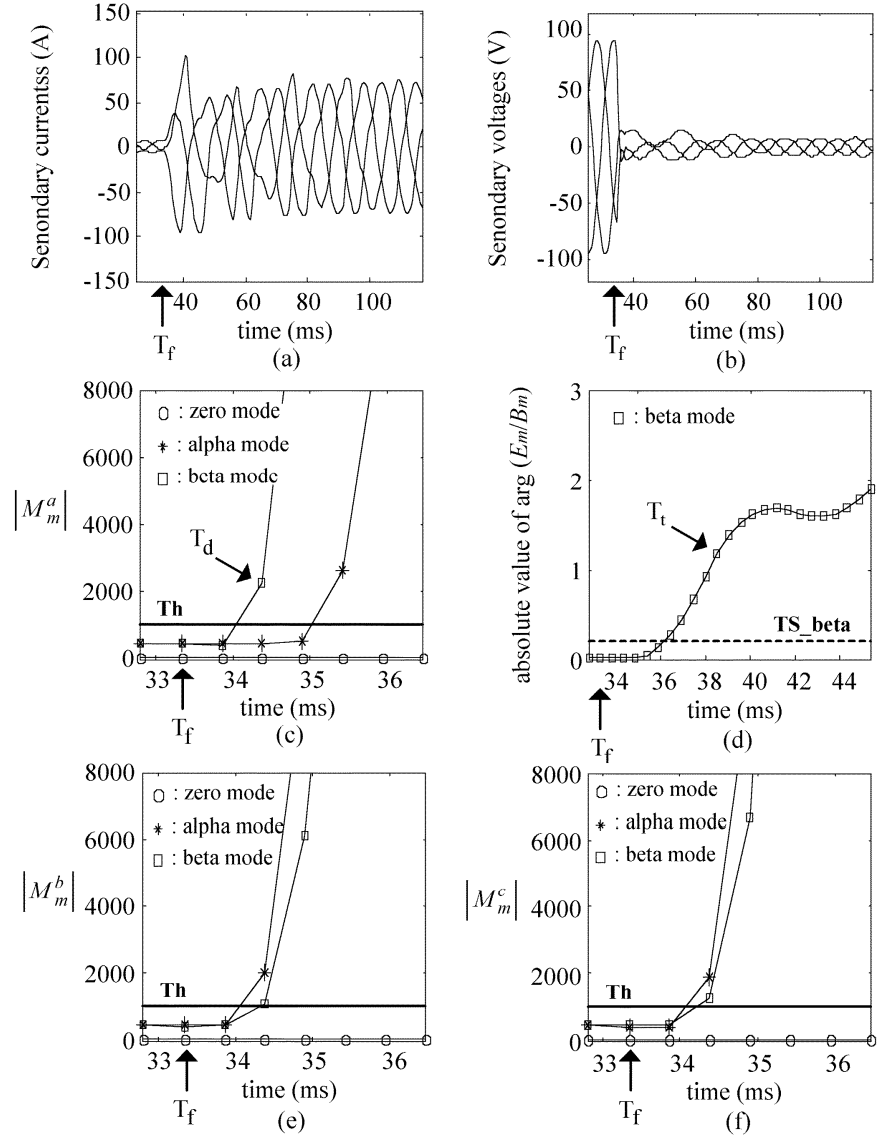

Fig. 9. Relay response for an internal a-b-c-g fault with CT saturation. (a) Three-phase current waveforms of sending end of the protected line. (b) Three-phase voltage waveforms of bus A. (c) The behaviors of modal fault detection index $\left|M_{m}\right|$ under phase-a basis. (d) The response curves of $\operatorname{abs}\left[\arg \left(E_{m} / B_{m}\right)\right]$. (e) and (f) are the behaviors of modal fault-detection index under phase-b and -c basis, respectively.

greater than the threshold value. So, the proposed scheme detects rapidly the external fault occurrence. Fig. 8(b) describes the response curves of abs $\left[\operatorname{angle}\left(E_{m} / B_{m}\right)\right]$ under this external fault event. Since the value of abs[angle $\left.\left(E_{m} / B_{m}\right)\right]$ is below the predefined threshold, the relaying scheme issues a restraint signal. Extensive simulation studies verify that the proposed scheme never misjudges any external fault events.

\section{Effects of CT Saturation and CVT Transients}

Extensive internal and external fault simulation studies are carried out to evaluate the effects of CT saturation and CVT transients on the relaying scheme. The selected result for an internal three-phase-to-ground fault is shown in Fig. 9. The fault location is set at 0.95 p.u. of the protected line. Fig. 9(a) and (b) show that CT saturation severely distorts the current waveforms and CVT transients affect the secondary voltages. As shown in Fig. 9(c), the beta-mode fault-detection index quickly detects the fault before the CT saturation. Fig. 9(d) shows the response curves of abs[angle $\left.\left(E_{m} / B_{m}\right)\right]$. The value of abs[angle $\left.\left(E_{m} / B_{m}\right)\right]$ is greater than the predefined threshold so the protection scheme correctly issues a tripping signal-to-circuit breaker under CT saturation. From Fig. 9(c), (e), and (f), the three-phase-to-ground fault is also correctly identified. The studies verify that the response of the proposed
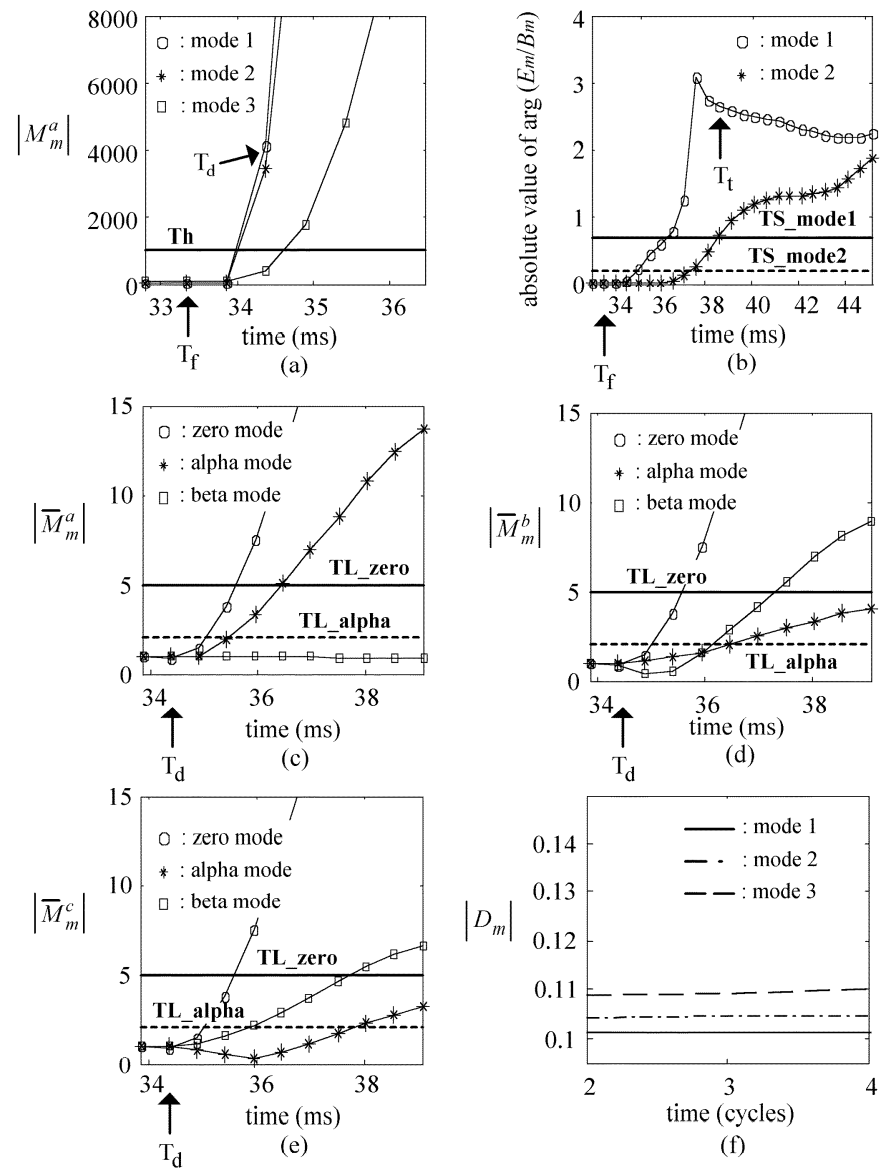

Fig. 10. The response of the protection scheme for untransposed lines. (a) The behaviors of modal fault-detection index $\left|M_{m}\right|$. (b) The response curves of $\operatorname{abs}\left[\arg \left(E_{m} / B_{m}\right)\right]$. (c)-(e) The behaviors of modified modal fault-detection index under phase-a, -b, and -c basis, respectively. (f) The response curves of modal fault-location index $\left|D_{m}\right|$. TS_mode1 $=0.7$ and TS_mode2 $=0.2$ are threshold settings for mode 1 and mode 2 signals, respectively. TL_zero $=5$ for zero mode and TL_alpha $=2$ for alpha and beta modes are threshold levels for modified modal detection index to perform fault classification and phase selection.

relaying scheme is unaffected by $\mathrm{CT}$ saturation and CVT transients under internal and external faults.

\section{Response Evaluation for Untransposed Lines}

To evaluate the performance of the proposed scheme for untransposed cases, the protected line $\mathrm{T} 1$ is modeled as an untransposed line with vertical configuration. A lot of fault studies, including different fault types, positions, and resistance have been conducted. An a-phase to ground fault is selected as the example to show the response performance of the complete protection scheme. The fault position is set at 0.1 p.u. and fault resistance is $1 \Omega$.

Fig. 10(a) and (b) shows the responses for the fault detection and direction discrimination. It is obviously seen that the scheme quickly detects the fault and the internal fault is confirmed at the time $5.208 \mathrm{~ms}$ after fault occurrence. Fig. 10(c)-(e) show the responses of the modified modal fault-detection index under different transformation basis. From these figures, when the internal fault is confirmed at the time $38.54 \mathrm{~ms}$, the fault type (ag) is also correctly identified. Fig. 10(f) shows the responses of modal fault-location index. The modal fault-location 


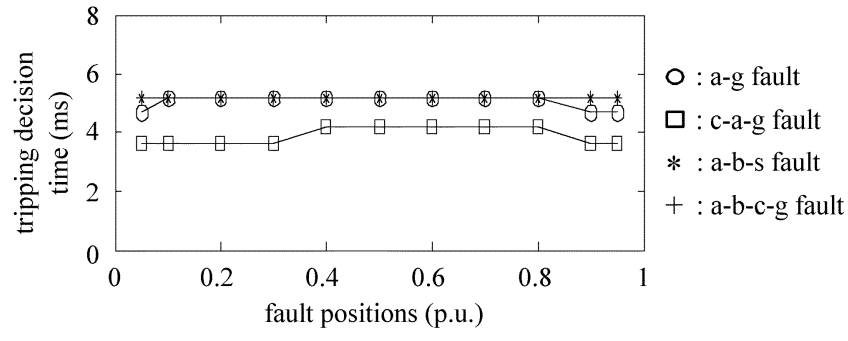

Fig. 11. Effects of fault types and positions on the tripping decision time.

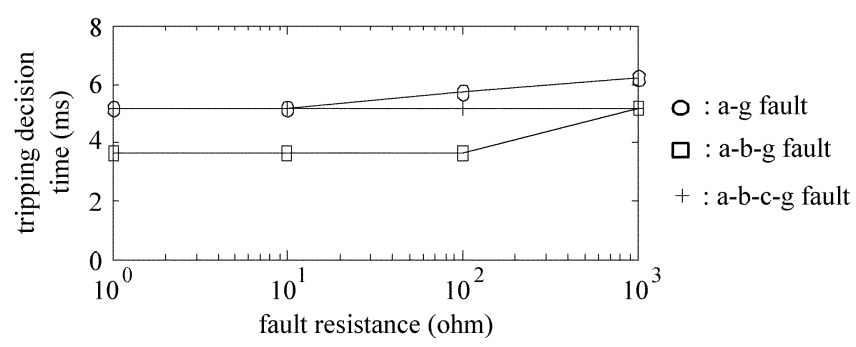

Fig. 12. Effects of fault-path resistance on the tripping decision time.

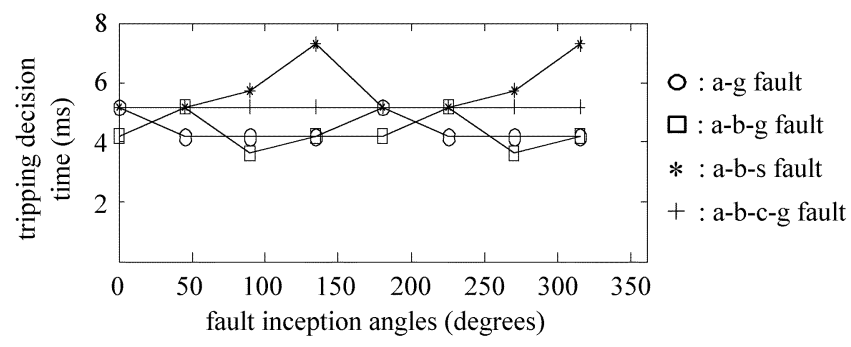

Fig. 13. Effects of fault inception angles on the tripping decision time.

errors are $0.13 \%, 0.44 \%$, and $0.92 \%$ for mode 1,2 , and 3 , respectively. They are all well below $1 \%$. The simulation studies have shown that the relaying performance is similar to that for transposed cases.

\section{E. Statistical Evaluation for Relay Response}

Due to limited space, only the results for transposed lines are given.

1) Different Fault Types and Positions: To examine the effects of different fault types and positions on the performance of the proposed relaying scheme, a lot of simulation studies have been conducted. The fault-path resistance is $1 \Omega$ and the selected results are shown in Fig. 11. It is clearly indicated that the proposed relaying scheme is almost insensitive to fault types and positions.

2) Different Fault-Path Resistance: A series of EMTP simulations with respect to different ground faults, various fault resistance, different fault locations, etc. has been conducted. Selected results are shown in Fig. 12. In these studies, fault locations are all set at 0.1 p.u. From the shown figure, we find that the proposed relaying technique is almost not affected, even the fault resistance is up to $1 \mathrm{k} \Omega$.

3) Different Fault Inception Angles: With respect to various fault types, selected simulation results are shown in Fig. 13. For comparison, fault positions of all tests are set at 0.8 p.u. and
TABLE II

Statistical Results of PeRformance EVAluation for Overall PROTECTION SCHEME

\begin{tabular}{|c|c|c|c|c|}
\hline $\begin{array}{r}\text { Evaluated } \\
\text { items }\end{array}$ & $\begin{array}{c}\text { Fault } \\
\text { fetection } \\
\text { types }\end{array}$ & $\begin{array}{c}\text { Tripping } \\
\text { decision }\end{array}$ & \multicolumn{2}{|c|}{$\begin{array}{c}\text { Fault location } \\
\text { error (\%) }\end{array}$} \\
\cline { 4 - 5 } & tims) & time (ms) & $\begin{array}{c}\text { Alpha } \\
\text { mode }\end{array}$ & $\begin{array}{c}\text { Beta } \\
\text { mode }\end{array}$ \\
\hline $\begin{array}{c}\text { Single phase to } \\
\text { ground fault }\end{array}$ & 2.150 & 4.892 & 0.406 & 0.362 \\
\hline $\begin{array}{c}\text { Double phase to } \\
\text { ground fault }\end{array}$ & 1.261 & 4.091 & 1.015 & 1.065 \\
\hline $\begin{array}{c}\text { Double phase } \\
\text { short fault }\end{array}$ & 2.043 & 5.920 & 1.005 & 0.867 \\
\hline $\begin{array}{c}\text { Three phase } \\
\text { fault }\end{array}$ & 1.042 & 5.351 & 0.961 & 0.477 \\
\hline Average & $\mathbf{1 . 6 2 4}$ & $\mathbf{5 . 0 6 3}$ & $\mathbf{0 . 8 4 7}$ & $\mathbf{0 . 6 9 3}$ \\
\hline
\end{tabular}

fault resistance is $1 \Omega$. The fault events are assumed to occur at points on waves for every $45^{\circ}$ spacing from 0 to $315^{\circ}$ refer to a-phase zero voltage point. It is clearly seen from Fig. 13 that the tripping decision time of the relaying scheme is not significantly affected by fault inception angles.

The performance evaluation associated with different source impedance and preload flows has also been conducted and the results are satisfactory. Due to limited space, these simulation results are not shown in this paper.

\section{F. Overall Performance of the Protection Scheme}

A total of 462 simulation studies have been conducted with respect to various system situations and fault events for transposed lines. The relaying scheme correctly detects all of the fault events and responds very fast. The statistical results are summarized in Table II. The average detection time for different fault types is $1.624 \mathrm{~ms}$. The complete relaying scheme on average takes $5.063 \mathrm{~ms}$ to make a tripping decision. As shown in Table II, the average fault-location error at the end of the third cycle after fault inception is well below $1 \%$.

In this work, the authors have also conducted 104 external fault tests, including various fault types, locations, and resistance. The proposed relaying scheme provides correct responses for all tested cases. This means that the relaying scheme provides excellent performance on dependability and security.

\section{CONCLUSIONS}

A new adaptive PMU-based protection scheme for EHV transposed/untransposed transmission lines is presented in this paper. The proposed scheme only uses a composite index to achieve high-speed relaying tasks including fault detection, direction discrimination, and classification. The scheme also provides accurate fault location.

Extensive simulation studies demonstrate that the complete protection scheme is not significantly affected by various system situations, fault events, and line configurations. The tripping decision time of the scheme is very fast and stable, whose value on average is well within half a cycle. The average fault-location error is less than $1 \%$. With the advancement of microprocessor and digital communication technology, the complete protection scheme is very feasible and effective for transmission-line protection. 


\section{REFERENCES}

[1] A. G. Phadke and J. S. Thorp, Computer Relaying for Power Systems. New York: Wiley, 1988.

[2] "Advancements in microprocessor based protection and communication," IEEE Tutorial Course, 1997.

[3] "Synchronized sampling and phasor measurements for relaying and control," IEEE Trans. Power Delivery, vol. 9, pp. 442-452, Jan. 1994.

[4] R. K. Aggarwal and A. T. Johns, "A differential line protection scheme for power systems based on composite voltage and current measurements," IEEE Trans. Power Delivery, vol. 4, pp. 1595-1601, July 1989.

[5] M. M. Mansour and G. W. Swift, "A multi-microprocessor based travelling wave relay-Theory and realization," IEEE Trans. Power Delivery, vol. PWRD-1, pp. 272-279, Jan. 1986.

[6] Z. Q. Bo, G. Weller, T. Lomas, and M. A. Redfern, "Positional protection of transmission systems using global positioning system," IEEE Trans. Power Delivery, vol. 15, pp. 1163-1168, Oct. 2000.

[7] M. Kezunović and B. Peruničić, "Automated transmission line fault analysis using synchronized sampling at two ends," IEEE Trans. Power Syst., vol. 11, pp. 441-447, Feb. 1996.

[8] H. Y. Li, E. P. Southern, P. A. Crossley, S. Potts, S. D. A. Pickering, B. R. J. Caunce, and G. C. Weller, "A new type of differential feeder protection relay using the global positioning system for data synchronization," IEEE Trans. Power Delivery, vol. 12, pp. 1090-1097, July 1997.

[9] J.-A. Jiang, J.-Z. Yang, Y.-H. Lin, C.-W. Liu, and J.-C. Ma, “An adaptive PMU based fault detection/location technique for transmission lines, Part I: Theory and algorithms," IEEE Trans. Power Delivery, vol. 15, pp. 486-493, Apr. 2000.

[10] J.-A. Jiang, Y.-H. Lin, J.-Z. Yang, T.-M. Too, and C.-W. Liu, "An adaptive PMU based fault detection/location technique for transmission lines, Part II: PMU implementation and performance evaluation," IEEE Trans. Power Delivery, vol. 15, pp. 1136-1146, Oct. 2000.

[11] IEEE Standard for Synchrophasors, IEEE Std. 1344-1995.

[12] H. W. Dommel, Electromagnetic Transients Programs-Reference Manual, 2nd ed. Vancouver, BC, Canada: Microtran Power System Analysis Corp., 1992.
Joe-Air Jiang (M'01) was born in Tainei, Taiwan, R.O.C., in 1963. He received the M.S. and Ph.D. degrees in electrical engineering from National Taiwan University, Taipei, Taiwan, R.O.C., in 1990 and 1999, respectively.

Currently, he is Assistant Professor of bio-industrial mechatronics engineering at National Taiwan University. From 1990 to 2001, he was with Private Kuang-Wu Institute of Technology and Commerce, Taipei, Taiwan, R.O.C. His areas of interest are in computer relaying, mechatronics, and bio-effects of electromagnetic wave.

Ching-Shan Chen (S'01) was born in Taichung, Taiwan, R.O.C., in 1976. He received the B.S. degree in electrical engineering from National Taiwan University of Science and Technology, Taipei, Taiwan, R.O.C., and the M.S. degree in electrical engineering from National Taiwan University (NTU), Taipei, in 1998 and 2000, respectively. He is currently pursuing the Ph.D. degree in electrical engineering at NTU.

His research includes computer relaying and the application of artificial intelligence to power system protection.

Chih-Wen Liu (S'93-M'96) was born in Taiwan in 1964. He received the M.S. and Ph.D. degrees in electrical engineering from Cornell University, Ithaca, NY, in 1992 and 1994, respectively.

Currently, he is Professor of electrical engineering at National Taiwan University, Taipei, Taiwan, R.O.C., where he has been since 1994. His main research area is in the application of computer technology to power system monitoring, operation, protection, and control.

Dr. Liu is a reviewer for IEEE TRANSACTIONS ON POWER SYSTEMS. 